\title{
Transient thermographic detection of buried defects: attempting to develop the prototype basic inspection procedure
}

\author{
by V. Vavilov1, S. Marinetti2, E. Grinzato2, P.G. Bison2, \\ I. Anoshkin1 and T. Kauppinen 3
}

${ }^{1}$ Tomsk Polytechnic University, Russia, 634028, Tomsk, 28, Savinykh St., 3; 2 ITEF-CNR, Corso Stati Uniti, 4, 35127-Padua, Italy; 3VTT Building Technology, P.O. Box 18021, FIN-90571, Oulu, Finland

\begin{abstract}
The transient thermal NDT basic inspection procedure is proposed. The procedure is based on the statistical analysis of NDT results presented as a single IR image or image sequence. A defect map of the sample under a test is created according to the accepted values of correct detection and false alarm. The proposed methodology could serve for comparing different thermal NDT procedures and data processing algorithms.
\end{abstract}

\section{Introduction}

Since the last QIRT-94 meeting some new trends have appeared in transient thermal NDT. One of focal points of interest is transferring laboratory techniques into workshop environment. This requires performing not only frontier research but also generalizing already available techniques and developing the so called basic inspection procedures (BIPs) [1]. This issue is supposed to be a hardcore of the coming "Handbook of Infrared NDT" which is being prepared by the ASNT [2].

We assume that a hypothetical BIP being applied to a sample to be inspected should finish at least with a specimen defect map (not speaking of defect characterization) which is to be made according to correct detection and false alarm requirements issued by the end-user of the NDT technique (figure 1). The BIP should include recommendations on both a hardware set-up and software package needed for performing an "optimum" thermal test. These issues are discussed in the present paper being forwarded, first of all, to the inspection of composites and layered structures.

\section{Step 1: Optimization of the test procedure}

A classical transient thermal NDT set-up includes a heater, an IR imager and a computer workstation. Location of the heater and the imager versus the specimen to be tested will specify one- or two-sided test. Heating could be flash, square-pulsed (or continuous) and periodic (harmonic). Furthermore, heating could be in a localized area (a point-by-point scanning scheme), in a line area (a line scanning scheme) or in a large-size area ("true" thermography). Conditionally, defects located closer to a front or rear surface, or in the middle of a specimen have to be analysed in a general case. Finally, at least three defect lateral size and three thickness values should be also considered to cover possible test variants. Therefore, altogether, the procedure optimization is to be made by $2 \times 3 \times 3 \times 3 \times 3 \times 3=486$ variants. It is recommended to use a numerical software, such as the "Termo. Heat" program from Tomsk Polytechnic University, to compile a Table of Test Variants where every variant will be specified with a maximum temperature signal over a defect together with its optimum observation time, as well as with needed heat power (allowed maximum specimen temperature is to be also taken into account). An example of such a table has been reported in fresco inspection [3].

\section{Step 2: Choice of the experimental set-up}

\subsection{The heater}

Data from the Table of Test Variants are to be confronted with peculiarities of a particular inspection procedure and hardware available. Below we shall specify some of these peculiarities. For example, in situ inspection of aluminum airframes for corrosion will definitely 
require a one-sided test where the area to be tested should be "black-painted" and heated within a large-size area or line. In the first case, the heating alternatives will be either a bank of flash tubes (flash heating) or a bank of quartz lamps (square-pulse heating). Within the line scanning scheme, a line quartz lamp moving over a surface shall be typically used. When inspecting carbon fiber reinforced plastic (CFRP) panels in a workshop environment, two-sided access could possibly be considered, and maximum specimen temperature could not typically exceed $100^{\circ} \mathrm{C}$. On the contrary, inspection of wall frescos would require only a one-sided test again, with maximum temperature increase not higher than $10^{\circ} \mathrm{C}$. This would require using of "soft" heaters, such as common electric bulbs, air fans etc. Flash tubes are expensive and able to deliver 1.5-3 kJ of electric energy each within typical 5-10 ms. Quartz lamps could be of 500-2000 W electric power and heat a specimen for tens of seconds. Point heating is able to provide a maximum temperature contrast over a defect but it is a seldom technique in industry because of low inspection productivity.

\subsection{The IR imager}

The most important parameters of IR imagers to be considered are as follows:

- temperature resolution $\left(0.05\right.$ to $\left.0.1^{\circ} \mathrm{C}\right)$

- image format, i.e. number of pixels and quantization level $(256 \times 256,12$ bit)

- view angle $\left(2.5 \times 2.5^{\circ}\right.$ to $\left.40 \times 40^{\circ}\right)$;

- frame frequency $(25 \mathrm{~Hz})$;

- a spectral band (2.5 to 5 and 8 to $14 \mu \mathrm{m})$;

- a type of the IR detector (SPRITE HgCdTe single or PtSi focal plane array).

The typical parameters are given in brackets. It is worth noticing that higher temperature resolution makes an image more friendly but not always improves defect detectivity. When inspecting high-conductive metals, focal plane array IR imagers are preferable since they provide simultaneous recording of all pixels in a frame.

Generally, a choice of an appropriate IR imager is not a difficult problem. in our research, we have used the Thermovision ${ }^{\mathrm{TM}}-900$ and Thermovision ${ }^{\mathrm{TM}}-550$ imagers manufactured by AGEMA (Sweden) which we found the most convenient for NDT purposes.

\subsection{The computer workstation}

This piece of hardware should provide acquisition of live images as a sequence of required length and format, with a lapse between consecutive images being determined using values of optimum observation times in the Table of Test Variants. For example, evaluation of corrosion in 1-3 mm steel samples is characterized with optimum observation times in the range from 0.2 to $1 \mathrm{~s}$. Therefore, in order to have a representative sequence, the acquisition interval should be 0.01-0.1 s. For example, a Thermovision ${ }^{\mathrm{TM}}-900$ model enables two recording modes with 15 or $30 \mathrm{~Hz}$ that results in 0.067 and $0.033 \mathrm{~s}$ lapse respectively. It is worth stressing that our inspection methodology is based on processing image sequences rather than single images. This enables data treatment in both space and time domains.

Versatility of modern computer workstations makes the problem of its choosing not difficult. In fact, a lot of modern research has been conducted using Silicon Graphics and Sun graphic stations. Data post-processing will be also successful with a Pentium.

\section{Step 3: Choice of the processing algorithm}

Within this step we omit use of standard image processing techniques which proved to be helpful but not decisive when treating IR thermograms. Filtration, look-up table transformation, $3 D$ viewing etc. are typically able to improve image appearance but do not increase a signalto-noise ratio, especially if noise comes mainly from a specimen surface. This conclusion may not concern Fourier-domain and morphological processing, but even these techniques have to be mostly used as the part of a more general algorithm based on heat conduction physics. Since this BIP step is directed toward optimization of a transient thermal NDT procedure in inspecting particular samples, to our opinion, it should be based on the experimental analysis of a reference specimen. 


\subsection{Sub-step 3.1: Creating the experimental sequence}

Transient thermal NDT data should be processed in both space and time domains to provide maximum detectivity of buried defects. The sequence to be analysed should include a specimen image at ambient temperature and a representative set of IR thermograms which correspond to heating-cooling process with potential defects being observable within it.

\subsection{Sub-step 3.2: Choosing the informative criterion}

This step assumes that, before inspecting a new sample, the decision making algorithm should be specified and verified using the reference specimen. The main feature of the reference specimen is that the operator knows location of defect and non-defect areas in it prior to a test.

Earlier, we have proposed to use the following informative criteria [3]: a temperature signal over a defect specified against a representative non-defect area $\Delta T(i, j, \tau)=T(i, j, \tau)-\bar{T}_{n d}(\tau)$, a heat transit time ${ }_{\tau_{\Delta T}^{*}}^{*}(i, j)$ defined as a specific time point in the $\Delta T(i, j, \tau)$ evolution, a normalized contrast $\Delta C(i, j, \tau)=C(i, j, \tau)-\bar{C}_{n d}(\tau)$, where $C(i, j, \tau)=T(i, j, \tau) / T(i, j, \tau$ ref $)$, and a respective heat transit time $\tau_{\Delta C}^{*}(i, j)$ defined as a specific time point in the $\Delta C(i, j, \tau)$ evolution. Notice that $T$ is excess temperature, and both the superscript " $k^{\prime \prime}$ and subscript "ref" specify a particular time in temperature evolution. The time $\tau^{*}$ is typically selected as a maximum signal time, or a half-rise time, or as the so called "eany detection time" [4]. The time $\tau_{\text {ref }}$ corresponds, as a rule, to the end of heating, having specified the so called normalization by a maximum temperature. The subscript "nd " means a non-defect area selected by the operator in the appropriate image of the reference specimen.

\subsection{Sub-step 3.3: Synthesizing images of false alarm, correct detection, signal-to-noise ratio and a defect map}

The proposed BIP has been developed to finish with a binary defect map which is to be created for accepted levels of false alarm $P_{f a}$. and correct detection $P_{c \cdot d}$. The $P_{f a}$. and $P_{c d}$. values will be determined for any pixel $(i, j)$ by analysing location of the single temperature value $T(i, j)$ within two histograms: of a non-defect and defect area. It is well known from statistics, that a partial area to the right from the $T(i, j)$ in a "non-defect histogram "represents the $P_{f . a}$. value. Respectively, a partial area to the left from the $T(i, j)$ in a "defect histogram "represents the $P_{c d}$. value. A specimen defect map will be made by assigning a binary value to the $(i, j)$-th pixel according to the following rule: $\operatorname{Amplitude}(i, j)=1$ if [ $P_{c d .}(i, j) \geq P_{c d .}^{n o r m}$ and $\left.P_{f a .}(i, j) \leq P_{f a .}^{n o r m}\right]$, otherwise Amplitude $(i, j)=0$.

The $P_{f a}=5 \%$ and $P_{c d . .}=80 \%$ could be recommended as the common normative values.

The Sub-step 3.3 is to be repeated for different procedures adopted from the Table of Test Variants, as well as for different informative criteria in order to recommend the "optimum" one. Afterwards, the obtained specimen defect map should be regarded as the best inspection result optimized by allowed test conditions.

\section{The heuristic technique for decision making}

The methodology above is based on applying known statistical procedures to a thermal NDT test. The parallel approach which relates more to heuristic operators' abilities when deciphering thermal images involves the so called Tanimoto criterion. Its use in the inspection of the CFRP specimen is described in [5]. The idea is that information on "false" indications and "missed" defects for a reference specimen is combined to create a single detectivity value which will reach $100 \%$ only if false alarm is zero and correct detection is unity.

However, the BIP methodology described in pp.2-4 is more formalized and, therefore, we believe it suits better an automated inspection. 


\section{Inspection of the CFRP specimen: the example of applying the BIP}

To demonstrate features of the proposed BIP, we have analysed the same sequence as in [4] obtained in the inspection of the $5 \mathrm{~mm}$ CFRP specimen which contained nine $80 \mu \mathrm{m}$ Teflon ${ }^{\mathrm{TM}}$ inserts sized from $4 \times 4$ to $12 \times 12 \mathrm{~mm}$ and located at depths from 0.25 to $2.5 \mathrm{~mm}$ (figure 2).

Step 1. Only a one-sided test has been studied. Since, by the time of this research, only two heaters were available - three flash tubes of $9 \mathrm{~kJ}$ total electric energy and sixteen quartz lamps of $32 \mathrm{~kW}$ total electric power - the numerical simulation was limited with the corresponding procedures. The defect $6 \times 6 \mathrm{~mm}$ at the depth $1.25 \mathrm{~mm}$ was selected in comparing different test procedures. Then, having used the "Termo.Heat" program, we found that the flash procedure which would involve only three flash tubes, will provide too low signal for the selected defect $\left(0.012^{\circ} \mathrm{C}\right.$ at $1.27 \mathrm{~s}$ for $5 \mathrm{~kJ}$ absorbed energy). Meanwhile, the squarepulse procedure was predicted to create the signal $1.07^{\circ} \mathrm{C}$ at $0.6 \mathrm{~s}$ for $20 \mathrm{~kW} / \mathrm{m}^{2}$ absorbed power after $5 \mathrm{~s}$ heating. The preliminary experiments proved that only the square-pulse test provided images of reasonable quality for defects located at $1.25 \mathrm{~mm}$ and deeper.

Step 2. The heater was selected as mentioned above. The LW Thermovision ${ }^{7 M}-900$ camera including the ERIKA software of AGEMA were used to record a sequence with the $1 \mathrm{~s}$ lapse. The Sun Sparc graphic station complemented with the "Termidge" software and the original statistical processing program have been used in data post-processing. The four typical source images from the sequence grabbed are shown in figure 2.

Step 3. The sequence to be evaluated was created according to the Sub-step 3.1. Both the temperature signal over defects and normalized contrast were analysed (Sub-step 3.2). The non-defect area chosen by the operator covered a whole surface of the specimen except nine "defect" areas. Nine defect areas which corresponded to nine true defects were used to create a "defect histogram". In order to analyse time evolution of statistical parameters, the pixel in the center of the defect $6 \times 6 \mathrm{~mm}$ at the depth $1.25 \mathrm{~mm}$ was selected. Evolution in time of both probabilities and signal-to-noise ratio is presented with graphs in figure 3a-c. It is well seen that image \#12 provides optimum inspection conditions. Both the histograms of this image shown in figure $4 a$ overlap each other. Probabilities values for the same image \#12 are presented in figure $4 b$ versus a pixel amplitude. Images of false alarm and correct detection are shown in figure $5 a, b$ (see also in colour). The final defect maps are presented in figure $5 c, d$ for two pairs of probabilities. These images illustrate clearly the test efficiency. It could be stated that use of the temperature signal criterion allows detecting of $6 \times 6 \mathrm{~mm}$ defects up to the depth $1.25 \mathrm{~mm}$ if to accept $P_{f a}=5 \%$ and $P_{c d . .}=80 \%$ (figure $5 \mathrm{c}$ ). If the end-user would be satisfied with $P_{f a .}=10 \%$ and $P_{c d . .}=50 \%$ values, the $12 \times 12 \mathrm{~mm}$ defect up to the depth $2.5 \mathrm{~mm}$ could be detected. Notice that we have deliberately omitted discussion on characterization of defect lateral size, depth and thermal resistance. For the same specimen this subject was studied in [6].

\section{Conclusion}

The proposed BIP gives a formalized basis for comparing different thermal NDT techniques and processing algorithms. Moreover, this procedure ends with a specimen defect map which is related to statistical parameters in decision making and, thus, is expressed in terms which are typically adopted by end-users of NDT techniques. Results which are produced with the BIP are typically less optimistic but more objective than heuristic conclusions made by operators.

\section{REFERENCES}

[1] DAVID (D.G.), MARTIN (J.Y.) and TRETOUT (H.R.). - Automatic defects recognition in composite aerospace structures from experimental and theoretical analysis as part of an intelligent infrared thermographic inspection system. Eklund (J.K.) ed., Thermosense-XIV, SPIE Proc. 1682, 1992, p.182-193.

[2] MACK (R.T.). - ASNT Thermal/Infrared Handbook: a new resource for testing service providers. Allen (L.R.) ed., Thermosense-XV, SPIE Proc. 1933, 1993, p.226-237.

[3] VAVILOV (V.P.), KOURTENKOV (D.G.), GRINZATO (E.), BISON (P.G.), MARINETTI (S.) and BRESSAN (C.). - Inversion of experimental data and thermal tomography using the "Termo.Heat" and "Termidge" software. In: Proc. of the Eurotherm Seminar 42 "Quantitative Infrared Thermography-QIRT'94", Aug.23-26, 1994, Sorrento, Italy, p.273-278. 
[4] KRAPEZ (J.-C). and BALAGEAS (D.). - Early detection of thermal contrast in pulsed stimulated infrared thermography. In: Proc. of the Eurotherm Seminar 42 "Quantitative Infrared Thermography-QIRT'94", Aug.23-26, 1994, Sorrento, Italy, p.260-266.

[5] VAVILOV (V.P.), BISON (P.G.), GRINZATO (E.). - Statistical evaluation of thermographic NDT performance applied to CFRP. Burleigh (D.) and Spicer (J.W.M.) eds., ThermosenseXVIII, SPIE Proc. 2766, 1996, p.174-177.

[6] GRINZATO (E.), VAVILOV (V.P.), BISON (P.G.), MARINETTI (S.) and BRESSAN (C.). Methodology of processing experimental data in transient thermal NDT. Semanovich (S.A.) ed., Thermosense-XVII, SPIE Proc. 2473, 1995, p.167-178.

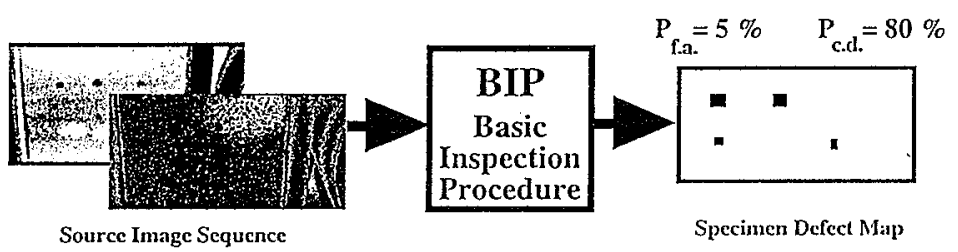

Fig.1. A basic inspection procedure in transient thermal NDT

Defects location

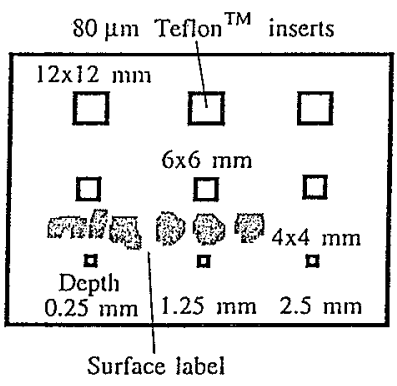

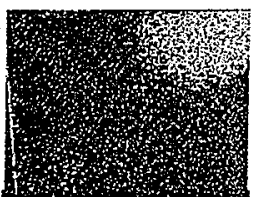

Ambient temperature

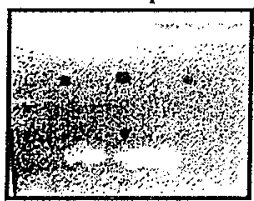

Optimum time

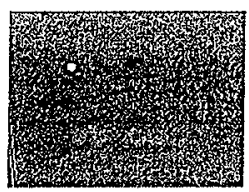

End of heating

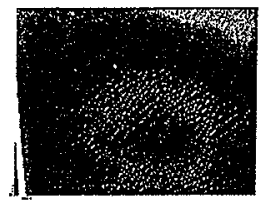

End of the process

Fig.2. Location of defects in the $5 \mathrm{~mm}$ CFRP specimen and the fragments of the image sequence

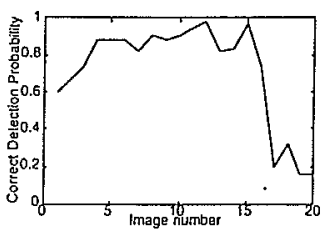

a)

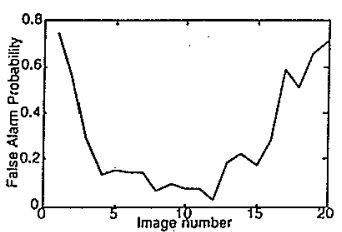

b)

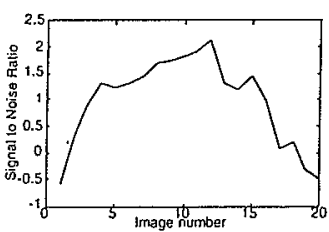

c)

Fig.3. Evolution of statistical parameters for the central pixe over the defect $6 \times 6 \mathrm{~mm}$ at the $1.25 \mathrm{~mm}$ depth:

a-probability of correct detection; b-probability of false alarm; c-signal-to-noise ratio 


\section{http://dx.doi.org/10.21611/qirt.1996.039}

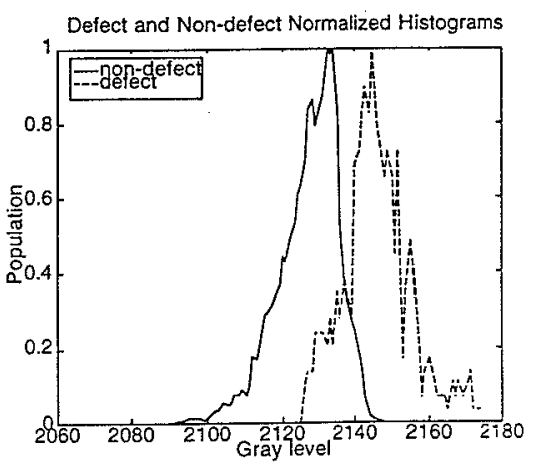

a)

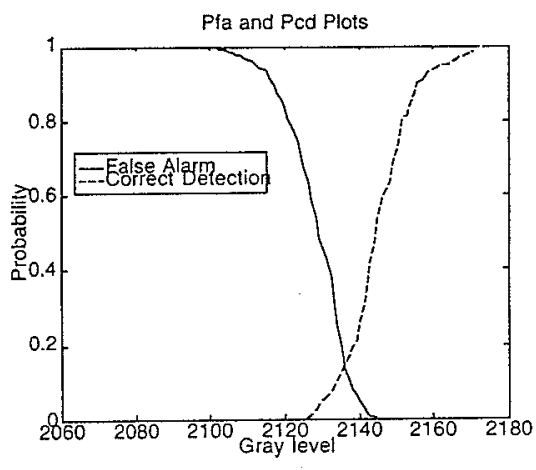

b)

Fig.4. Statistical distributions of pixel amplitudes for "non-defect" and "defect" areas (a) and cumulative probabilities of correct detection and false alarm (b)

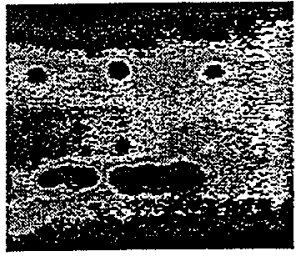

a)

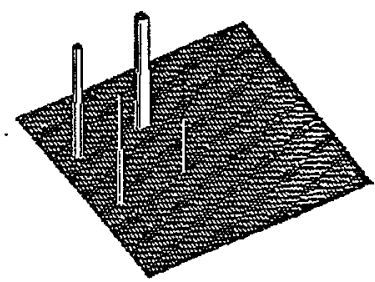

c)

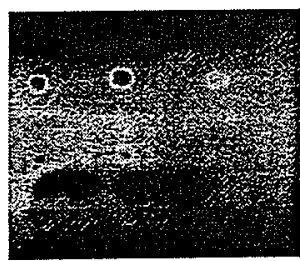

b)

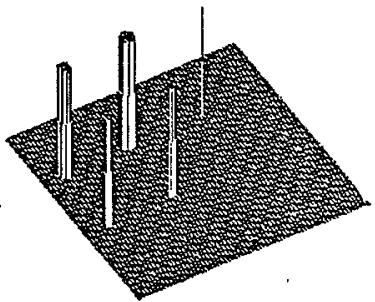

d)

Fig.5. Statistical evaluation of the "best" image in the sequence (the $5 \mathrm{~mm}$ CFRP specimen of Fig.2; see also in colour):

a-image of false alarm $P_{f}$ a :

b-image of correct detection $P_{c . d}$-specimen defect map for $P_{\text {f. }}=5 \%$ and $P_{c . c}=80 \%$; d-specimen defect map for $P$ f.a: $10 \%$ and $P_{c . d}=50 \%$ 